\title{
A Compressive Damage Constitutive Model for Rock Mass with a Set of Nonpersistently Closed Joints under Biaxial Conditions
}

\author{
Hongyan Liu ${ }^{1,2}$ and Xiaoping Yuan ${ }^{3}$ \\ ${ }^{1}$ College of Engineering \& Technology, China University of Geosciences (Beijing), Beijing 100083, China \\ ${ }^{2}$ School of Engineering, Tibet University, Lhasa, Xizang 850000, China \\ ${ }^{3}$ Laboratoire de Géologie, CNRS, Ecole Normale Supérieure, 24 rue Lhomond, 75005 Paris, France
}

Correspondence should be addressed to Hongyan Liu; lhyan1204@126.com

Received 6 October 2014; Revised 9 March 2015; Accepted 11 March 2015

Academic Editor: Chaudry Masood Khalique

Copyright (C) 2015 H. Liu and X. Yuan. This is an open access article distributed under the Creative Commons Attribution License, which permits unrestricted use, distribution, and reproduction in any medium, provided the original work is properly cited.

\begin{abstract}
One of the most problems faced by the practical rock engineering is to evaluate the rock mass strength. Now the existing theoretical evaluation of the mechanical property of jointed rock mass has no satisfactory answer yet because of the great number of variables involved. One of them is the nonpersistent joints which inherently affect the rock mass mechanical behavior. The previous models for rock mass can only reflect the effect of joint geometrical property on its mechanical behavior. Accordingly, this paper presents a new theoretical model to evaluate the mechanical behavior of the rock mass with a set of nonpersistently closed joints under biaxial conditions, which can reflect the effect of both the joint geometrical and mechanical property on the mechanical behavior of the rock mass under biaxial conditions at the same time. A series of calculation examples validate that the proposed model is capable of presenting the effect of joint geometrical and mechanical properties and the confining pressure on rock mass strength at the same time.
\end{abstract}

\section{Introduction}

Existing in many rock mass projects, such as rock slopes, open pits, and underground caverns, joints have significant effects on the rock mass mechanical behaviors, such as its strength, deformability, failure mode, and stability, which will directly influence the design and construction of the rock mass engineering [1-3]. The existence of the nonpersistent joints and their interaction under the far-field stresses often lead to high stress concentration at the tips of the joint and become the source of weakening and failure of the rock mass [4, 5]. Jennings [6] proposed to compute the combined strength of joint and rock bridges from the simple linear weighing of the strength contributed by each fraction of material:

$$
\tau=k\left(c_{j}+\sigma \tan \phi_{j}\right)+(1-k)\left(c_{r}+\sigma \tan \phi_{r}\right)
$$

where $\left(c_{j}, \phi_{j}\right)$ and $\left(c_{r}, \phi_{r}\right)$ represent the cohesion and friction angle of the joint and of the intact rock, respectively, and $k$ is the joint continuity factor given by

$$
k=\frac{L_{j}}{\left(L_{j}+L_{r}\right)},
$$

where $L_{j}$ and $L_{r}$ are the length of the joint and of the rock bridge, respectively.

Equation (1) disregards the influence of the joints on the stress distribution, and assumes simultaneous failure of the intact material and the joints.

Different procedures have been used to study the strength of rock mass with nonpersistent joints: field observations, numerical studies or laboratory tests, and analytical solutions. All these methods have their own advantages and disadvantages. Among them, the analytical solution develops quickly in recent years with the widespread application of mechanical and mathematical tools in the study of the rock 
mass mechanical behavior. Now the following three analytical approaches are often used to study the constitutive relation of a jointed rock mass. The first is the phenomenological approach based on continuum damage mechanics $[7,8]$, in which the effects of microscopic damage mechanisms on property of the rock mass are reflected by scalar, vector, or tensor damage variables. For example, Kawamoto et al. [9] and Swoboda et al. [10] both adopted the second-order tensor to describe the damage anisotropy in the rock mass caused by the joints. Kawamoto et al. [9] presented a damage model for the jointed rock mass with one set of joints:

$$
\Omega^{k}=\frac{l}{V} S^{k}\left(n^{k} n^{k}\right)
$$

where $\Omega^{k}$ is the damage tensor caused by the joints to the rock mass, $S^{k}$ is the size of the jointed area, $n^{k}$ is the unit normal vector to the joint surface, $l$ is the average spacing of the joints, and $V$ is the volume of the rock mass.

For $N$-set of joints, the damage tensor is given as the sum over all the related damage tensor obtained from (3):

$$
\Omega=\frac{l}{V} \sum_{k=1}^{N} S^{k}\left(n^{k} n^{k}\right) .
$$

It is a common method to define the damage variable in rock mass geometrical damage theory [9-11]. But its deficiency is obvious, in which only the geometrical property of the joint such as its length and dip angle is included, while the mechanical property of the joint such as its shear strength, namely, its cohesion and internal friction angle, is not included. That is to say, the above damage variable definition method thinks that the damage cannot transfer the stress, which is nearly true for the rock mass under tension, but not for the rock mass under compression. This is because the joint will close and then slide along its surface under compression, in which the joint will transfer part of the compressive and shear stresses. And the transferring coefficients are much related to the mechanical property of the joint such as its internal friction angle and cohesion [10]. Therefore, many scholars began to adopt different methods to improve the above model. When the rock mass is subjected to compression, Kawamoto et al. [9] and Yuan et al. [12] introduced the joint transferring coefficients of the compressive and shear stresses to revise the above model according to the fact that the joint can transfer part of the compressive and shear stresses. However, how to accurately obtain these two coefficients also becomes a new problem. While Swoboda and Yang [13] introduced the material constant $H_{d}(0 \leq$ $\left.H_{d}<1\right)$ to consider the effect of joint closure on the stress transferring, now it is obtained mainly by experience.

The second approach is based on mesomechanical damage mechanics, which leads to an improved understanding of the underlying physical process. In this approach, the nucleation, growth, and coalescence of microcracks are studied and their influences on mechanical properties are reflected in the constitutive relation in certain ways [14]. To study the mechanical behaviors of a joint-weakened rock mass by the micromechanical approach, several micromechanicsbased joint models, such as the cylindrical pore model
[15], dislocation pile-up model [16], and the frictional sliding crack model, have been proposed. Among these models, the frictional sliding crack model is widely accepted [17-20].

The third is the phenomenological approach based on mesomechanical mechanics, developed in recent years, which lies between the above two approaches and combines the advantages of them. It has a certain application in rock mechanics study [21].

In sum, it can be seen from the existing studies that the above three approaches all have been used in the study of the damage constitutive model for the jointed rock mass. And it is widely accepted that the geometrical property of the joint such as its length, dip angle, and number and the mechanical property such as its internal friction angle and cohesion should be both included in the rock mass constitutive model, only by which the effect of the joint on rock mass mechanical behavior is objectively reflected. But these two kinds of properties of the joint such as the geometrical ones and mechanical ones are separately considered in the existing study. Namely, the geometrical property of the joint is firstly adopted to define the damage tensor, and then its mechanical property such as its shear strength are adopted to revise the above calculation result, which not only causes inconvenience in application of this model but also is prone to lead to much error in the calculation result because of the arbitrariness in selecting these parameters. Can a damage tensor which includes both geometrical and mechanical property of the joint be proposed? This kind of damage tensor is not only in good agreement with the failure mechanism of the jointed rock mass but also applicable to use, which can avoid the error caused by the selection of the parameters to a large extent. So some research works have been done on this subject. For instance, Li et al. [22] obtained the calculation method of the mesodamage tensor of the rock mass with nonpersistently closed joints based on the strain energy theory, which perfectly considers the joint geometrical and mechanical property at the same time. It provides a good idea for studying the damage mechanics of the rock mass with nonpersistent joints. However, they only study the jointed rock mass under uniaxial condition, while in practical engineering, the rock mass is always in the complicated stress conditions such as biaxial or triaxial conditions. Arora [23] made triaxial compressive tests on specimens of plaster of Paris containing a single joint, and the result showed that increase in the lateral pressure leads to a more isotropic behavior. The study done by Prudencio and van Sint Jan [24] also illustrated that the confining stress resulted in different failure modes and higher peak strength of the jointed specimens. Therefore, it is very important and necessary to study the mechanical behavior of the rock mass under the complicated stress conditions.

Overall, the existing models provided a basis for estimating the mechanical behavior of the rock mass. Yet, since only a part is involved in the existing models, further key factors should be taken into account for the sophisticated mechanical behavior of the rock mass as follows: 
(1) the major property of the joints, such as its shear strength, can be further considered in the calculation of the damage tensor;

(2) the effect of the complicated stress conditions such as the biaxial stress condition on the rock mass mechanical behavior should be studied in order to perfectly solve the practical engineering problem.

As such, the present work aims to establish a mathematical model to consider the above-mentioned factors.

The establishment of the proposed model is presented. Next, the calculation examples are done to check its validity and to further demonstrate the characteristics of the proposed model. The proposed model considers the major factors of joints to provide a mean in determining the mechanical behavior of the rock mass with a set of nonpersistently closed joints under biaxial conditions.

\section{Establishment of the Damage Model for the Rock Mass with Nonpersistently Closed Joints}

According to fracture mechanics, for a planar problem under biaxial conditions, the increment of the additional strain energy $U_{1}$ because of the existence of the joints is as follows (the third stress intensity factor $K_{\mathrm{III}}=0$ in the planar problem):

$$
U_{1}=\int_{0}^{A} G d A=\frac{1}{E^{\prime}} \int_{0}^{A}\left(K_{\mathrm{I}}^{2}+K_{\mathrm{II}}^{2}\right) d A,
$$

where $G$ is the energy release rate, $A$ is the joint area, and $K_{\mathrm{I}}$ and $K_{\mathrm{II}}$ are the first and second stress intensity factors of the joint tip, respectively. For a planar strain problem, $E^{\prime}=E$, and for a planar stress problem $E^{\prime}=E /\left(1-v^{2}\right)$, where $E$ and $\nu$ are Young's elastic modulus and Poisson's ratio of the corresponding intact rock, respectively. Because the planar stress problem is studied in this paper, $E^{\prime}=E$ is adopted.

For a single joint, $A=B a$ (unilateral joint) or $2 B a$ (central joint). For many joints, $A=N B a$ (unilateral joint) or $2 \mathrm{NBa}$ (central joint), where $N$ is the joint number, $B$ is the joint depth, and $a$ is the joint half length, as shown in Figure 1.

For the rock mass with nonpersistent joints (shown in Figure 1) under the biaxial stresses $\sigma_{1}$ and $\sigma_{3}\left(\sigma_{2}=0\right.$ is adopted in the biaxial conditions), its damage strain energy $Y$ is

$$
Y=-\frac{\sigma_{\mathrm{eq}}{ }^{2}}{2 E(1-D)^{2}}\left\{\frac{2}{3}(1+\nu)+3(1-2 \nu)\left(\frac{\sigma_{m}}{\sigma_{\mathrm{eq}}}\right)^{2}\right\},
$$

where $\sigma_{m}=(1 / 3)\left(\sigma_{1}+\sigma_{3}\right)$ is the mean stress, $\sigma_{\text {eq }}=$ $(1 / \sqrt{2}) \sqrt{\sigma_{1}^{2}+\left(\sigma_{1}-\sigma_{3}\right)^{2}+\sigma_{3}^{2}}$ is Mises effective stress, $D$ is the damage variable, and $\sigma_{1}, \sigma_{2}$, and $\sigma_{3}$ are the first, second, and third principle stresses, respectively.

$U^{E}$ is the unit volume elastic strain energy corresponding to a certain stress, and, under the biaxial conditions, it can be expressed as

$$
U^{E}=-(1-D) Y \text {. }
$$

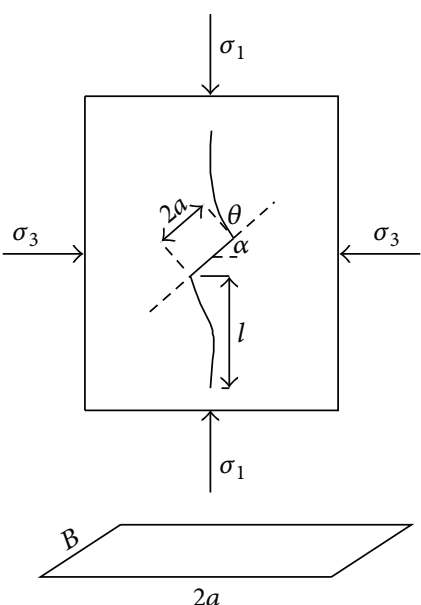

FIGURE 1: Model of nonpersistently jointed rock mass: joint length $2 a$, joint dip angle $\alpha$, growing crack length $l$, and joint depth $B$.

Substituting (6) into (7), we obtain

$$
U^{E}=-\frac{\sigma_{\mathrm{eq}}^{2}}{2 E(1-D)}\left\{\frac{2}{3}(1+\nu)+3(1-2 \nu)\left(\frac{\sigma_{m}}{\sigma_{\mathrm{eq}}}\right)^{2}\right\} \text {. }
$$

When the rock mass does not contain any joints, $D=0$, and (8) can be changed into

$$
U_{0}^{E}=-\frac{\sigma_{\mathrm{eq}}^{2}}{2 E}\left\{\frac{2}{3}(1+\nu)+3(1-2 \nu)\left(\frac{\sigma_{m}}{\sigma_{\mathrm{eq}}}\right)^{2}\right\} .
$$

The increment of the unit volume elastic strain energy caused by the joint is

$$
\begin{aligned}
\Delta U^{E}= & U^{E}-U_{0}^{E} \\
= & \left(\frac{\sigma_{\mathrm{eq}}{ }^{2}}{2 E(1-D)}-\frac{\sigma_{\mathrm{eq}}{ }^{2}}{2 E}\right) \\
& \cdot\left\{\frac{2}{3}(1+\nu)+3(1-2 \nu)\left(\frac{\sigma_{m}}{\sigma_{\mathrm{eq}}}\right)^{2}\right\} .
\end{aligned}
$$

Assuming the volume of the rock mass is $V$, the increment of the elastic strain energy caused by the existence of the joint is

$$
\begin{aligned}
\Delta U^{E}= & V\left(\frac{\sigma_{\mathrm{eq}}{ }^{2}}{2 E(1-D)}-\frac{\sigma_{\mathrm{eq}}{ }^{2}}{2 E}\right) \\
& \cdot\left\{\frac{2}{3}(1+\nu)+3(1-2 \nu)\left(\frac{\sigma_{m}}{\sigma_{\mathrm{eq}}}\right)^{2}\right\} .
\end{aligned}
$$

$\Delta U^{E}$ in (11) and $U_{1}$ in (5) are both the increment of the elastic strain energy caused by the joint, and they should be equal to each other:

$$
\Delta U^{E}=U_{1}
$$


or

$$
\begin{aligned}
\frac{1-\nu^{2}}{E} & \int_{0}^{A}\left(K_{\mathrm{I}}^{2}+K_{\mathrm{II}}^{2}\right) d A \\
= & V\left(\frac{\sigma_{\mathrm{eq}}^{2}}{2 E(1-D)}-\frac{\sigma_{\mathrm{eq}}^{2}}{2 E}\right) \\
& \cdot\left\{\frac{2}{3}(1+\nu)+3(1-2 \nu)\left(\frac{\sigma_{m}}{\sigma_{\mathrm{eq}}}\right)^{2}\right\} .
\end{aligned}
$$

From (13), we obtain

$$
\begin{aligned}
D=1-\left(1+\frac{2}{V} \frac{\left(1-\nu^{2}\right)}{\sigma_{\mathrm{eq}}^{2}\left\{(2 / 3)(1+\nu)+3(1-2 \nu)\left(\sigma_{m} / \sigma_{\mathrm{eq}}\right)^{2}\right\}}\right. \\
\left.\cdot \int_{0}^{A}\left(K_{\mathrm{I}}^{2}+K_{\mathrm{II}}^{2}\right) d A\right)^{-1} .
\end{aligned}
$$

Then SIF $K_{\mathrm{I}}$ and $K_{\mathrm{II}}$ can be obtained by analyzing the mechanical behavior of the jointed rock mass.

\subsection{SIF Calculation of a Single Nonpersistently Closed Joint} Tip in Rock Mass. Under the biaxial compression, the normal and shear stresses will both occur on the joint surface of the rock mass. The normal stress will make the joint close and the shear stress will make the rock mass have the trend of sliding along the joint surface. Because of the closure of the joint, the direction of the friction force is opposite to that of the sliding. When the shear stress exceeds the friction one on the joint surface, the rock mass will slide. With the increase in compressive load, the wing cracks will begin to propagate from the joint tips at the direction of $70.5^{\circ}$ [25-27]; namely, the joints will propagate along the direction, in which the tensile stress approaches its maximum, as shown in Figure 1. The formation of the wing cracks is caused by the tensile stress at the joint tips because of the friction slide along the joint surface.

Under the biaxial compression, the normal and shear stresses on the joint surface are as follows, respectively:

$$
\begin{gathered}
\sigma_{\alpha}(\sigma, \alpha)=\sigma_{1} \cos ^{2} \alpha+\sigma_{3} \sin ^{2} \alpha, \\
\tau_{\alpha}(\sigma, \alpha)=\frac{\sigma_{1}-\sigma_{3}}{2} \sin 2 \alpha,
\end{gathered}
$$

where $\sigma(\sigma, \alpha)$ and $\tau(\sigma, \alpha)$ are the normal and shear stresses on the joint surface, respectively and $\alpha$ is the joint dip angle.

Assume the joint internal friction angle is $\varphi$, and accordingly the friction coefficient $\mu$ of the joint surface is $\tan \varphi$. Then under the biaxial compression, the shear stress will cause the rock block to slide along the joint surface. In turn, the normal stress on the joint surface will produce the friction one to resist the slippage of the rock block along the joint surface. So the slide force $\tau_{\text {eff }}$ along the joint surface must be more than or equal to zero and cannot be less than zero. Therefore, $\tau_{\text {eff }}$ can be obtained from (15):

$$
\tau_{\mathrm{eff}}= \begin{cases}0 & \tau_{\alpha}<\mu \sigma_{\alpha} \\ \tau_{\alpha}-\mu \sigma_{\alpha} & \tau_{\alpha} \geq \mu \sigma_{\alpha}\end{cases}
$$

It is noted that the joint cohesion is neglected in (16), which indicates that the method proposed in this paper is suitable for the rock mass whose cohesion is zero or little and can be neglected.

The SIF $K_{\mathrm{I}}$ and $K_{\mathrm{II}}$ of the wing cracks at the joint tips can be revised as follows according to Lee [28] and the propagation direction of the wing cracks:

$$
\begin{gathered}
K_{\mathrm{I}}=-\frac{2 a \tau_{\mathrm{eff}} \sin \theta}{\sqrt{\pi\left(l+l^{*}\right)}}+\sigma_{\alpha+\theta}(\boldsymbol{\sigma}, \alpha+\theta) \sqrt{\pi l}, \\
K_{\mathrm{II}}=-\frac{2 a \tau_{\mathrm{eff}} \cos \theta}{\sqrt{\pi\left(l+l^{*}\right)}}-\tau_{\alpha+\theta}(\boldsymbol{\sigma}, \alpha+\theta) \sqrt{\pi l},
\end{gathered}
$$

where $\sigma_{\alpha+\theta}$ and $\tau_{\alpha+\theta}$ can be obtained from (15), $a$ is the joint half length, $l$ is the propagation length of wing cracks, and $\theta$ is the propagation angle of the wing cracks at the joint tips, and it is assumed to be $70.5^{\circ}[25,26] . l^{*}=0.27 a$ was introduced [29] to make $K_{\mathrm{I}}$ and $K_{\mathrm{II}}$ nonsingular when the tensile joint length is small.

A parameter $l^{*}(=0.27 a)$ was added to obtain proper behavior at the short wing crack limit. One can observe in (17a) and (17b) that as the crack extends, that is, as $l$ increases, the driving force, that is, $K_{\mathrm{I}}$, diminishes drastically indicating crack arrest. However, in reality, the crack grows, subsequently interacting and coalescing with adjoining flaws. These micromechanical processes result in macroscopic axial splitting, which are the typical brittle failure mode observed under compression in brittle solids. Hence, the present models are not able to completely capture the physics of the phenomenon of compressive failure in brittle solids. The reasons for this could be attributed to several factors that include (i) the assumption of uniform shear stress along the joint surfaces during the crack propagation and (ii) the neglect of local heterogeneity in obtaining stress fields.

Considering the critical condition of the wing crack to propagate, namely, the propagation length of the wing cracks $l=0$, the $K_{\mathrm{I}}$ and $K_{\mathrm{II}}$ of the wing cracks are

$$
\begin{gathered}
K_{\mathrm{I}}=-\frac{2 a \tau_{\mathrm{eff}} \sin \theta}{\sqrt{\pi l^{*}}}, \\
K_{\mathrm{II}}=-\frac{2 a \tau_{\mathrm{eff}} \cos \theta}{\sqrt{\pi l^{*}}} .
\end{gathered}
$$

It can be known from above that the condition that the wing crack length $l=0$ is the initial condition that the nonpersistently closed joint does not propagate. If the SIF of the joint tip at this moment is solved, the initial damage of the rock mass caused by the original nonpersistently closed joint can be obtained from (14). It is obviously seen that the damage obtained from this method includes not only the 


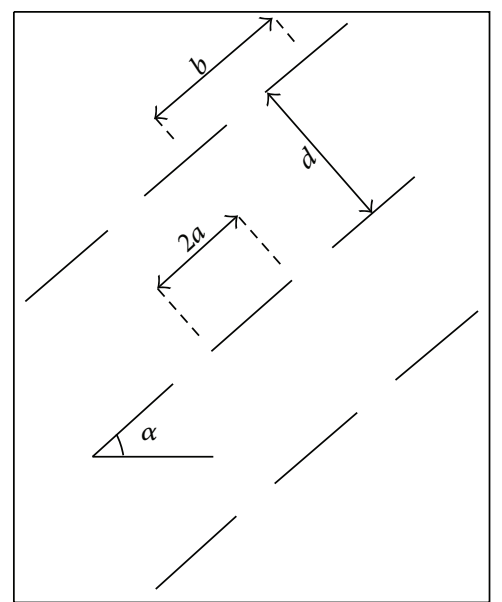

FIGURE 2: Model of nonpersistently jointed rock mass.

joint geometrical property such as its length and dip angle but also its mechanical property such as the internal friction angle. So the damage constitutive model for the jointed rock mass obtained with this method is in more agreement with the actual condition, in which the joint transferring coefficients of the compressive and shear stresses are not required to revise it.

2.2. Multijoints in One Line or Grouped in Lines. If there is a group of joints on the same line with the same length and interval in the analysis region, the stress intensity factor can be written as

$$
\begin{gathered}
K_{\mathrm{I}}=K_{\mathrm{I} 0} \sqrt{\frac{2}{\pi \phi} \tan \frac{\pi \phi}{2},} \\
K_{\mathrm{II}}=K_{\mathrm{II} 0} \sqrt{\frac{2}{\pi \phi} \tan \frac{\pi \phi}{2}},
\end{gathered}
$$

where $K_{\mathrm{I} 0}$ is the stress intensity factor for a single joint under tension condition, $K_{\mathrm{I}}$ is the stress intensity factor for the group joints, and so on, and $\phi=2 a / b$ is the joint area ratio to the area of the whole section.

If there is a group parallel line of joints as in Figure 2, then the interactions of the joints can be expressed as follows:

$$
\begin{aligned}
K_{\mathrm{I}} & =f(a, b, d) K_{\mathrm{I} 0}, \\
K_{\mathrm{II}} & =f(a, b, d) K_{\mathrm{II} 0} .
\end{aligned}
$$

Under the assumption of the joints shown in Figure 2, Cherepanov [30] proposed the interaction factor of the group joints as in Table 1 . The damage variables for the single set of grouped joints can then be derived.

2.3. The Damage Variable of Rock Mass with One Set of Joints. When the rock mass contains a set of one-rowed
TABLE 1: $f(a, b, d)$ value.

\begin{tabular}{lccccc}
\hline$d / 2 a$ & & \multicolumn{5}{c}{$b / 2 a$} \\
& $\infty$ & 5.0 & 2.5 & 1.67 & 1.25 \\
\hline$\infty$ & 1.0 & 1.017 & 1.075 & 1.208 & 1.565 \\
5 & 1.016 & 1.020 & 1.075 & 1.208 & 1.565 \\
1 & 1.257 & 1.257 & 1.258 & 1.292 & 1.580 \\
0.25 & 2.094 & 2.094 & 2.094 & 2.094 & 2.107 \\
\hline
\end{tabular}

nonpersistently closed joints, it can be obtained by substituting (15) (16) and (18a) and (18b) (19a) and (19b) into (14):

$$
D=\left\{\begin{array}{cr}
0 & \tau_{\alpha}<\mu \sigma_{\alpha} \\
1-\left(1-\frac{7.56 B N a^{2}}{V \phi^{2}}\right. & \\
\cdot \tau_{\mathrm{eff}}{ }^{2} \ln \left(\cos \frac{\pi \phi}{2}\right) & \left(\sigma _ { \mathrm { eq } } ^ { 2 } \left\{\frac{2}{3}(1+\nu)\right.\right. \\
\left.\left.\left.+3(1-2 \nu)\left(\frac{\sigma_{m}}{\sigma_{\mathrm{eq}}}\right)^{2}\right\}\right)^{-1}\right)^{-1} & \tau_{\alpha} \geq \mu \sigma_{\alpha} .
\end{array}\right.
$$

When the rock mass contains a set of more-rowed nonpersistently closed joints, it can be obtained by substituting (15) (16) and (18a) and (18b) and (20a) and (20b) into (14):

$$
D=\left\{\begin{array}{cc}
0 & \tau_{\alpha}<\mu \sigma_{\alpha} \\
1-\left(1+\frac{18.86 B N a^{2}}{V} f^{2}(a, b, d)\right. & \\
\cdot \tau_{\mathrm{eff}}^{2}\left(\sigma _ { \mathrm { eq } } ^ { 2 } \left\{\frac{2}{3}(1+\nu)\right.\right. & \\
\left.\left.\left.+3(1-2 \nu)\left(\frac{\sigma_{m}}{\sigma_{\mathrm{eq}}}\right)^{2}\right\}\right)^{-1}\right)^{-1} \\
\tau_{\alpha} \geq \mu \sigma_{\alpha},
\end{array}\right.
$$

where $N$ is the joint number, $V$ is the volume of the rock mass sample, and the other parameters are as above.

2.4. Tensor of the Damage Variable. Because of the singularity of the macroscopic damage, $D$ obtained from (21) or (22) is only the damage along the vertical direction. It is necessary to make it tensorial to reflect the anisotropy of the macroscopic damage. The tensorial methods are many, and here the method proposed by Kawamoto et al. [9] is adopted by introducing damage tensor $\Omega$. Let $\Omega=D \omega$, where $\omega$ is the second-order symmetrical tensor. The calculation method of $\omega$ is as follows. For the rock mass with one set of nonpersistently closed joints, assume the angle between the 


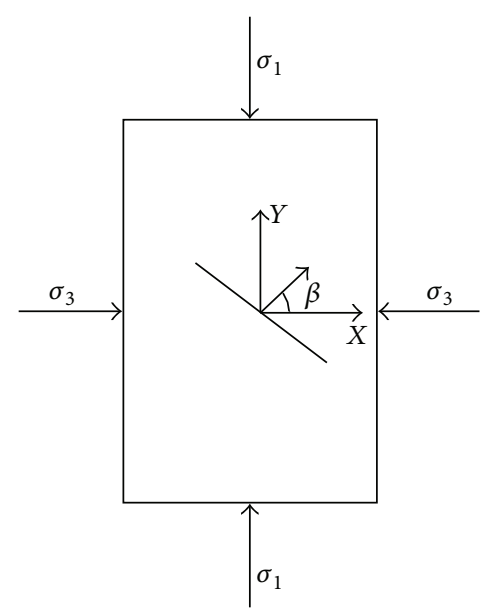

FIGURE 3: The mechanical model of jointed rock mass.

normal direction of the joint and $x$ axis is $\beta$, shown in Figure 3 and the unit normal vector of the joint is $n, \omega$ is given by:

$$
\omega=n \otimes n=\left[\begin{array}{cc}
\cos ^{2} \beta & \sin \beta \cos \beta \\
\cos \beta \sin \beta & \sin ^{2} \beta
\end{array}\right] .
$$

\section{Analysis of Calculation Examples}

3.1. The Mechanical Behavior of Rock Mass with One Single of Nonpersistently Closed Joint. Figure 1 is the calculation model that is $10 \mathrm{~cm}$ high and $5 \mathrm{~cm}$ wide. The geometrical parameters $B$ and $a$ are $1 \mathrm{~cm}$ and $2 \mathrm{~cm}$, respectively. The joint internal friction angle is $30^{\circ}$ and its cohesion is assumed to be zero. The climax strength, Young's elastic modulus, and Poisson's ratio of the corresponding intact rock are $80 \mathrm{MPa}, 20 \mathrm{GPa}$, and 0.25 , respectively. Then its damage variable $D$ according to this proposed model is

$$
D=\left\{\begin{array}{c}
0 \\
1-\left(1+\frac{9.43 B a^{2}}{V} r \mu \sigma_{\alpha}\right. \\
\cdot \tau_{\mathrm{eff}}^{2}\left(\sigma _ { \mathrm { eq } } ^ { 2 } \left\{\frac{2}{3}(1+\nu)\right.\right. \\
\left.\left.\left.+3(1-2 v)\left(\frac{\sigma_{m}}{\sigma_{\mathrm{eq}}}\right)^{2}\right\}\right)^{-1}\right)^{-1} \\
\tau_{\alpha} \geq \mu \sigma_{\alpha}
\end{array}\right.
$$

because the damage variable $D$ of the jointed rock mass can also be defined as

$$
D=1-\frac{\sigma_{j}}{\sigma_{r}}
$$

where $\sigma_{j}, \sigma_{r}$ are the climax strength of the jointed rock mass and the corresponding intact rock.

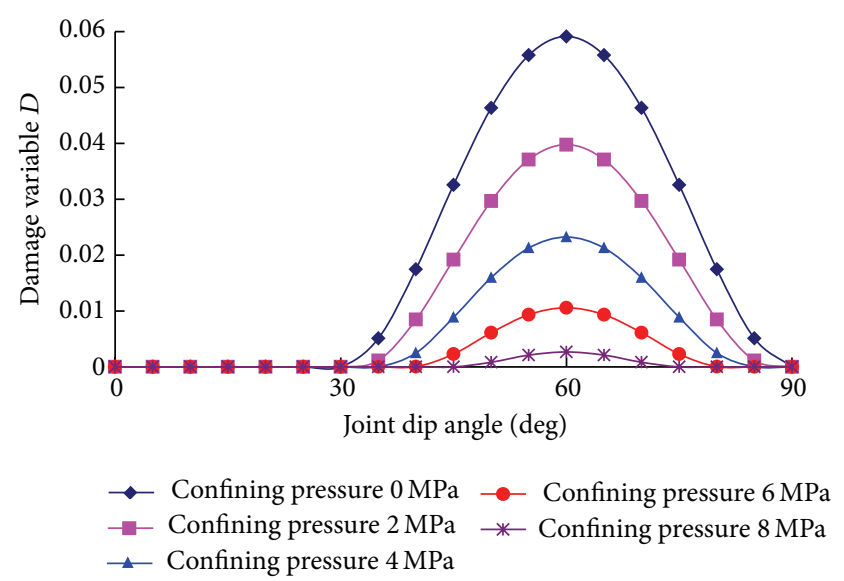

(a) The change law of the sample's damage variable with the joint dip angle under the different confining pressure

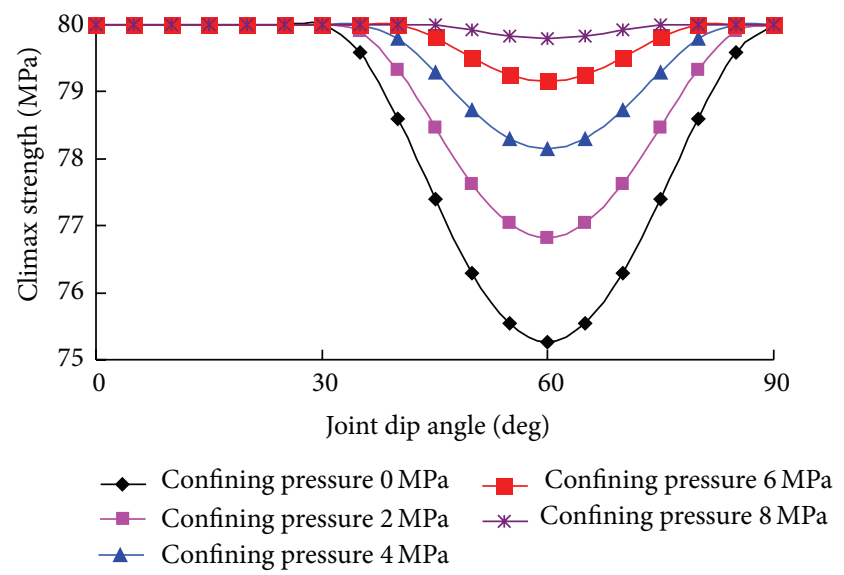

(b) The change law of the sample's climax strength with the joint dip angle under the different confining pressure

FIgURE 4: The effect of confining pressure on rock mass damage variable and climax strength.

If we assume that $\sigma_{1}$ is $30 \mathrm{MPa}$, then the change laws of the rock mass damage variable and climax strength with the joint dip angle under different confining pressure are shown in Figure 4.

It can be seen from Figure 4 that the change laws of rock mass damage variable and climax strength with the joint dip angle are parabolas with the hatch up and down, respectively. And the rock mass climax strength is the least and its damage variable is the maximal when the joint dip angle is about $60^{\circ}$. The rock mass strength is the same as that of the intact rock and the damage variable is zero when the joint dip angle lies between a certain scope, which fits with that obtained by Prudencio and van Sint Jan [24]. With increase in confining pressure, the sample's damage variable decreases and climax strength increases. That is to say the confining pressure can heighten the sample's climax strength and reduce its damage.

Then the effect of the joint half-length on the rock mass damage variable is discussed. Assume the confining pressure is $4 \mathrm{MPa}$ and the other parameters are as stated above; Figure 5 shows the rock mass damage variable versus the 


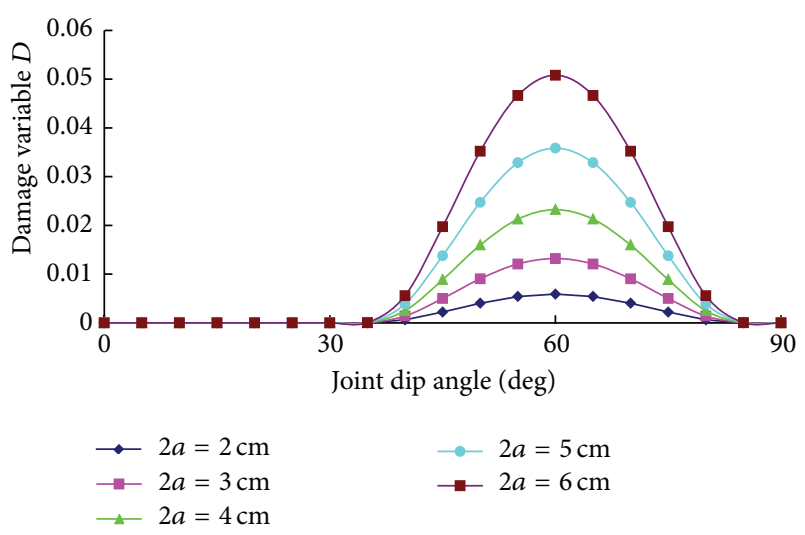

FIGURE 5: The rock mass damage variable versus the joint length.

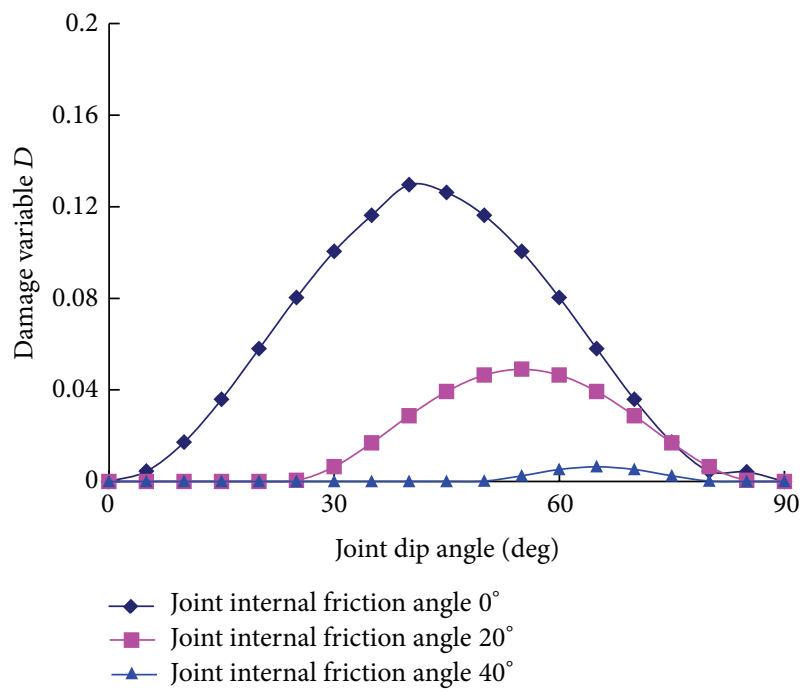

FIGURE 6: The rock mass damage variable versus the joint internal friction angle.

joint half-length. It can be seen that the rock mass damage variable increases with the increase in joint half-length, and when the joint half-length is little, the increment rate of the damage is rather less, but when the joint half-length is large, the increment rate of the damage variable is much more.

Then the effect of joint internal friction angle on the rock mass damage variable is discussed. Assume the joint halflength is $4 \mathrm{~cm}$, confining pressure is $4 \mathrm{MPa}$, and the other parameters are as stated above; the rock mass damage variable versus the joint internal friction angle is shown in Figure 6. It can be seen that the rock mass damage variable rapidly decreases with the increase in joint internal friction angle. This is because the joint shear strength increases with the joint internal friction angle. Correspondingly the sample's climax strength increases and its damage variable decreases. Meanwhile, with the increase in joint internal friction angle, the joint dip angle correspondent to the maximum damage variable of the sample increases correspondingly. When the joint dip angles are $0^{\circ}, 20^{\circ}$, and $40^{\circ}$, respectively, the corresponding joint dip angles are $45^{\circ}, 55^{\circ}$, and $65^{\circ}$, respectively.

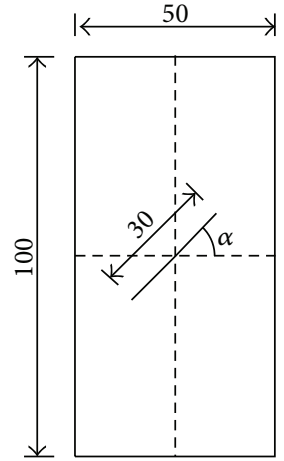

(a) one joint

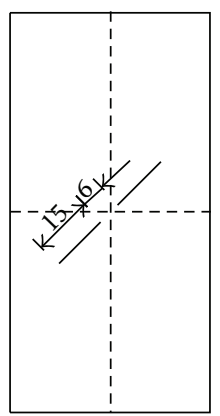

(b) two joints

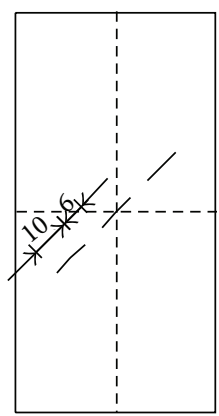

(c) three joints

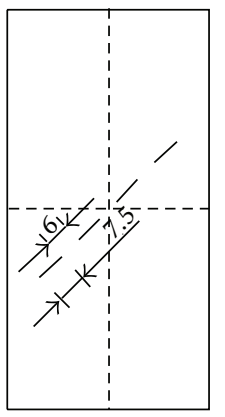

(d) four joints

Figure 7: Models of rock mass with joints of same length but different number (unit: $\mathrm{mm}$ ).

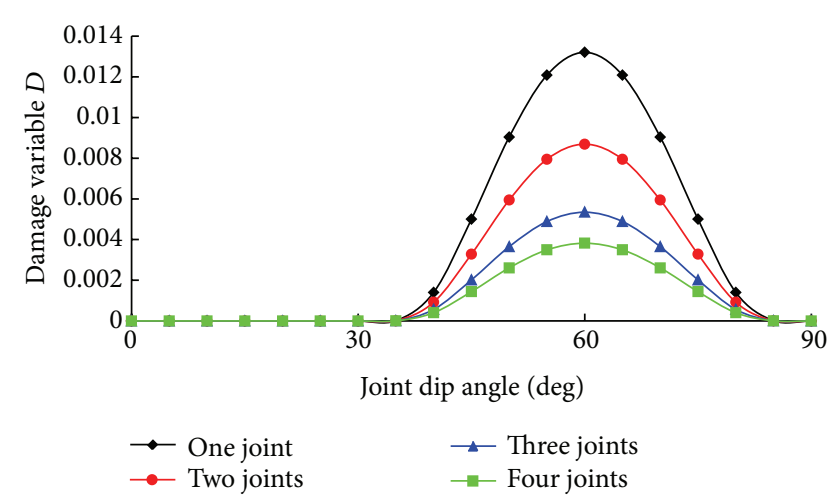

FIgURE 8: The rock mass damage variable versus the joint number.

3.2. The Mechanical Property of Rock Mass with One Row of Nonpersistently Closed Joints in One Set. Take the rock mass with the joint whose total length is the same but the number is different as an example, and the calculation models are shown in Figure 7. The joint total length in Figures $7(\mathrm{a}) \sim 7(\mathrm{~d})$ is all $30 \mathrm{~mm}$, but the joint number is $1,2,3$, and 4 , respectively. The intervals between the two neighboring joints are all $6 \mathrm{~mm}$. Assume $\sigma_{1}$ and $\sigma_{3}$ are $30 \mathrm{MPa}$ and $4 \mathrm{MPa}$, respectively. The joint internal friction angle is $30^{\circ}$. The Young's elastic modulus and Poisson's ratio of the corresponding intact rock are $20 \mathrm{GPa}$ and 0.25 , respectively. The rock mass damage variable versus the joint number is shown in Figure 8. 
It can be seen from Figure 8 that (1) when the joint total length is the same, the rock mass damage variable gradually decreases with the increase in the joint number, and the rock mass strength gradually increases. This can be explained with fracture mechanics. With the increase in the joint number, the number of joint tips increases; namely, when there is one joint, there are only two joint tips, and when there are four joints, there are eight joint tips. It is known from fracture mechanics that the energy for the joint initiation is far more than that for the joint propagation. So, the number of the joint initiation point increases with the increase in the joint number, and correspondingly the energy for the joint initiation greatly increases, which will cause the rock mass damage to decrease and its climax strength to increase. (2) From the decrement rate of the damage variable, the decrease in rock mass damage is not linear with the increase in the joint number. For this calculation example, the rock mass damage increases when the joint number increases from one to two are much less than that when the joint number increases from two to three, which indicates that there exists the complicated interaction among the joints. And it may have some relationships with the geometrical and mechanical property of the joints. (3) The results show that the long joint should not be formed in rock mass slope reinforcement engineering or it should be interrupted with anchoring or grouting, while in rock fragmentation engineering such as blasting and cutting, one or more main joints should be formed as soon as possible in order to make the rock mass fail.

\subsection{The Mechanical Property of Rock Mass with Multirows of} Nonpersistently Closed Joints in One Set. Here the effect of the row interval of joints on rock mass mechanical behavior is studied, and the calculation model is shown in Figure 9. This model has four joints in two rows and one set. Here the parameters of $a$ and $b$ are $2 \mathrm{~cm}$ and $5 \mathrm{~cm}$, respectively. The joint row interval $d$ is $1 \mathrm{~cm}, 4 \mathrm{~cm}$, and $20 \mathrm{~cm}$, and $d / 2 a$ are $0.25,1$, and 5 , respectively. Assume $\sigma_{1}$ and $\sigma_{3}$ are $30 \mathrm{MPa}$ and $4 \mathrm{MPa}$, respectively. The joint internal friction angle is $30^{\circ}$. The Young's elastic modulus and Poisson's ratio of the corresponding intact rock are $20 \mathrm{GPa}$ and 0.25 , respectively. The rock mass damage variable versus the row interval of joints is shown in Figure 10.

It can be seen from Figure 10 that (1) the rock mass damage variable gradually decreases with the increase in row interval of joints, and the rock mass strength gradually increases. It can be known from fracture mechanics that the stress concentration will occur at the joint tips when the sample is loaded. But the region of the stress concentration is limited, which is mainly located near the joint tips. Therefore, when the joints are near enough, the overlap of the stress fields at the joint tips will cause the rock mass to be seriously damaged and the strength to decrease, while with the increase in the row interval of joints, the interaction among the stress fields of the joint tips will decrease and eventually completely disappear, and the rock mass damage will gradually increase and tend to be a certain value. (2) For the decrement rate of the damage variable, the rock mass damage firstly decreases

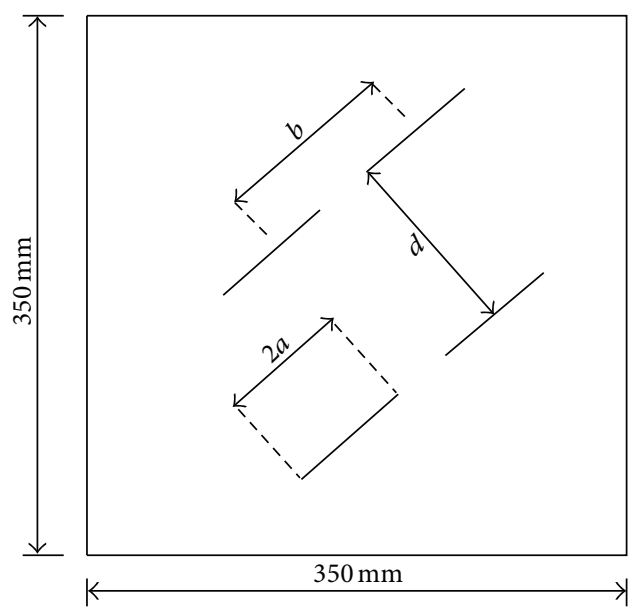

FIGURE 9: Rock mass model with two rows of joints in one set.

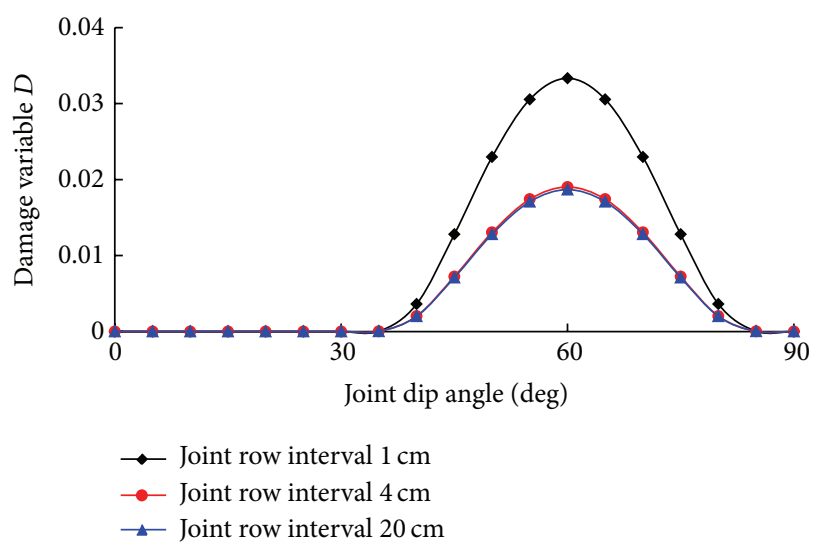

FIGURE 10: The rock mass damage variable versus the row interval of joints.

rapidly and then tends to be a constant with the increase in row interval of joints. Take this calculation example for instance, the maximal damage variable of the rock mass rapidly decreases from 0.033 to 0.019 with the increase in row interval of joints from $1 \mathrm{~cm}$ to $4 \mathrm{~cm}$, and its decrement extent reaches $42.4 \%$. However, the maximal damage variable of the rock mass rapidly decreases from 0.019 to 0.0187 with the increase in row interval of joints from $4 \mathrm{~cm}$ to $20 \mathrm{~cm}$, and its decrement extent is only $1.58 \%$. It indicates that the interaction among the joints can be neglected when the row interval of joints becomes large enough.

\section{Conclusions}

After discussing the damage and fracture evolution mechanism of the rock mass with the nonpersistently closed joints under biaxial condition, the following main conclusions are drawn.

(1) Aiming at the shortcoming that only the joint geometrical property is included in the most existing jointed rock mass damage constitutive models while the joint shear strength is not, a new definition method of the damage 
variable for the jointed rock mass by comprehensively considering the joint geometrical and mechanical properties is proposed. It is hopeful to provide a new path for studying the damage mechanics of the jointed rock mass.

(2) For the rock mass with a single nonpersistently closed joint, the rock mass climax strength is the minimal and its damage variable is the maximal when the joint dip angle is about $60^{\circ}$. It agrees with the existing research conclusions, which indicates the validity of this proposed model.

(3) The proposed model is adopted to discuss the effect of joint length and internal friction angle on rock mass damage. The result shows that the rock mass damage increases nonlinearly with the increase in joint length, while it decreases nearly proportional to the increase in joint internal friction angle.

(4) For the rock mass with a set of one-rowed nonpersistently closed joints, when the joint total length is the same, the rock mass damage gradually decreases with the increase in the joint number, but the decrement rate is not linear with it. It indicates that the complicated interaction among the joints exists.

(5) For the rock mass with a set of multirowed nonpersistently closed joints, the rock mass damage firstly decreases rapidly and then tends to be constant with the increase in row interval of joints. It indicates that the interaction among joints mainly exists in a small region near the joint tips and it will gradually weaken with the increase in the row interval of joints and even disappear.

\section{Conflict of Interests}

The authors declare that there is no conflict of interests regarding the publication of this paper.

\section{Acknowledgment}

This study is supported by the Fundamental Research Funds for the Central Universities (2-9-2014-019) of China, Ministry of Education Science, and Technology Key Research Funds (211175) of China and National Natural Science Funds (41002113 and 41162009) of China.

\section{References}

[1] M. Akin, "Slope stability problems and back analysis in heavily jointed rock mass: a case study from Manisa, Turkey," Rock Mechanics and Rock Engineering, vol. 46, no. 2, pp. 359-371, 2013.

[2] T. T. Wang and T. H. Huang, "Anisotropic deformation of a circular tunnel excavated in a rock mass containing sets of ubiquitous joints: theory analysis and numerical modeling," Rock Mechanics and Rock Engineering, vol. 47, no. 2, pp. 643657, 2014.

[3] H. Bejari and J. Khademi Hamidi, "Simultaneous effects of joint spacing and orientation on TBM cutting efficiency in jointed rock masses," Rock Mechanics and Rock Engineering, vol. 46, no. 4, pp. 897-907, 2013.

[4] L. N. Lens, E. Bittencourt, and V. M. R. d'Avila, "Constitutive models for cohesive zones in mixed-mode fracture of plain concrete," Engineering Fracture Mechanics, vol. 76, no. 14, pp. 2281-2297, 2009.

[5] S. Marfia and E. Sacco, "A fracture evolution procedure for cohesive materials," International Journal of Fracture, vol. 110, no. 3, pp. 241-261, 2001.

[6] J. E. Jennings, "A mathematical theory for the calculation of the stability of open cast mines," in Proceedings of the Symposium on Theoretical Background to the Planning of Open Pit Mines, pp. 87-102, Johannesburg, South Africa, 1970.

[7] J. A. Hudson and S. D. Priest, "Discontinuity frequency in rock masses," International Journal of Rock Mechanics and Mining Sciences and, vol. 20, no. 2, pp. 73-89, 1983.

[8] M. Oda, "Similarity rule of crack geometry in statistically homogeneous rock masses," Mechanics of Materials, vol. 3, no. 2, pp. 119-129, 1984.

[9] T. Kawamoto, Y. Ichikawa, and T. Kyoya, "Deformation and fracturing behavior of discontinuous rock mass and damage mechanics theory," International Journal for Numerical and Analytical Methods in Geomechanics, vol. 12, no. 1, pp. 1-30, 1988.

[10] G. Swoboda, X. P. Shen, and L. Rosas, "Damage model for jointed rock mass and its application to tunnelling," Computers and Geotechnics, vol. 22, no. 3-4, pp. 183-203, 1998.

[11] D. Zhao, G. Swoboda, and F. Laabmayr, "Damage mechanics and its application for the design of an underground theater," Tunnelling and Underground Space Technology, vol. 19, no. 6, pp. 567-575, 2004.

[12] H.-P. Yuan, P. Cao, and W.-Z. Xu, "Mechanism study on subcritical crack growth of flabby and intricate ore rock," Transactions of Nonferrous Metals Society of China, vol. 16, no. 3, pp. 723-727, 2006.

[13] G. Swoboda and Q. Yang, "An energy-based damage model of geomaterials-I. Formulation and numerical results," International Journal of Solids and Structures, vol. 36, no. 12, pp. 17191734, 1999.

[14] X. P. Zhou, Q. Ha, Y. X. Zhang, and K. S. Zhu, "Analysis of deformation localization and the complete stress-strain relation for brittle rock subjected to dynamic compressive loads," International Journal of Rock Mechanics and Mining Sciences, vol. 41, no. 2, pp. 311-319, 2004.

[15] J. X. Zhang, T. F. Wong, and D. M. Davis, "Micromechanics of pressure-induced grain crushing in porous rocks," Journal of Geophysical Research, vol. 95, no. B1, pp. 341-352, 1990.

[16] T. F. Wong, "A note on the propagation behavior of a crack nucleated by a dislocation pileup," Journal of Geophysical Research, vol. 95, no. 6, pp. 8639-8646, 1990.

[17] M. F. Ashby and S. D. Hallam, "The failure of brittle solids containing small cracks under compressive stress states," Acta Metallurgica, vol. 34, no. 3, pp. 497-510, 1986.

[18] A. Brencich and L. Gambarotta, "Isotropic damage model with different tensile-compressive response for brittle materials," International Journal of Solids and Structures, vol. 38, no. 34-35, pp. 5865-5892, 2001.

[19] H. B. Li, J. Zhao, and T. J. Li, "Micromechanical modelling of the mechanical properties of a granite under dynamic uniaxial compressive loads," International Journal of Rock Mechanics and Mining Sciences, vol. 37, no. 6, pp. 923-935, 2000.

[20] S. Li, W. Zhu, W. Chen, and J. Xu, "Mechanical model of multi-crack rock mass and its engineering application," Acta Mechanica Sinica, vol. 16, no. 4, pp. 357-365, 2000. 
[21] X. P. Yuan, H. Y. Liu, and Z. Q. Wang, "An interacting crackmechanics based model for elastoplastic damage model of rocklike materials under compression," International Journal of Rock Mechanics and Mining Sciences, vol. 58, no. 3, pp. 92-102, 2013.

[22] N. Li, W. Chen, P. Zhang, and G. Swoboda, "The mechanical properties and a fatigue-damage model for jointed rock masses subjected to dynamic cyclical loading," International Journal of Rock Mechanics and Mining Sciences, vol. 38, no. 7, pp. 1071-1079, 2001.

[23] V. K. Arora, Strength and deformation behavior of jointed rocks [Ph.D. thesis], Indian Institute of Technology, 1987.

[24] M. Prudencio and M. van Sint Jan, "Strength and failure modes of rock mass models with non-persistent joints," International Journal of Rock Mechanics and Mining Sciences, vol. 44, no. 6, pp. 890-902, 2007.

[25] C. Huang and G. Subhash, "Influence of lateral confinement on dynamic damage evolution during uniaxial compressive response of brittle solids," Journal of the Mechanics and Physics of Solids, vol. 51, no. 6, pp. 1089-1105, 2003.

[26] C. Huang, G. Subhash, and S. J. Vitton, "A dynamic damage growth model for uniaxial compressive response of rock aggregates," Mechanics of Materials, vol. 34, no. 5, pp. 267-277, 2002.

[27] B. Paliwal and K. T. Ramesh, "An interacting micro-crack damage model for failure of brittle materials under compression," Journal of the Mechanics and Physics of Solids, vol. 56, no. 3, pp. 896-923, 2008.

[28] S. Lee and G. Ravichandran, "Crack initiation in brittle solids under multiaxial compression," Engineering Fracture Mechanics, vol. 70, no. 13, pp. 1645-1658, 2003.

[29] H. Horii and S. Nemat-Nasser, "Brittle failure in compression: splitting, faulting and brittle-ductile transition," Philosophical Transactions of the Royal Society of London, vol. 319, no. 1549, pp. 337-374, 1986.

[30] G. P. Cherepanov, Mechanics of Brittle Fracture, McGraw-Hill, New York, NY, USA, 1977. 


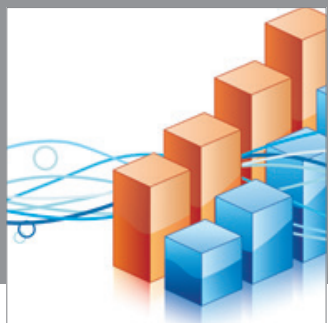

Advances in

Operations Research

mansans

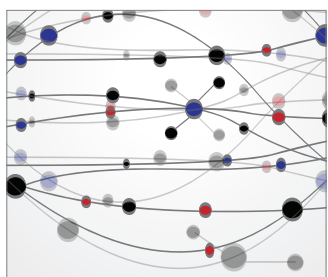

The Scientific World Journal
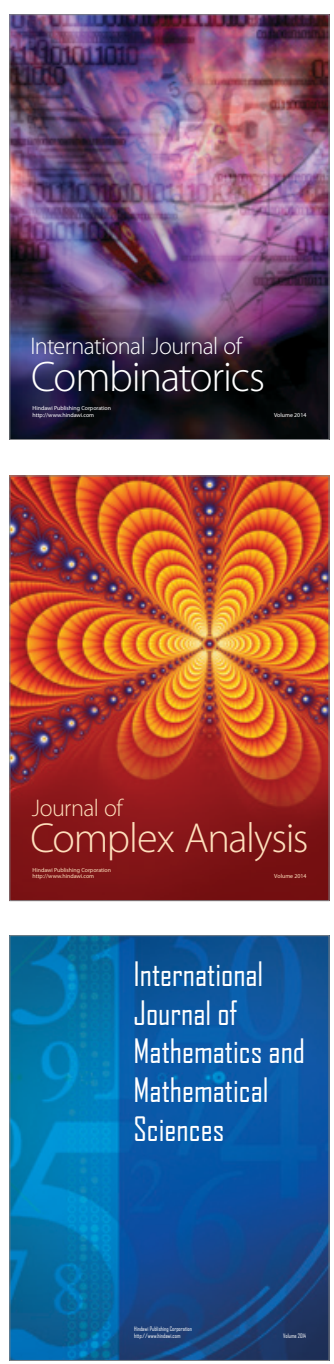
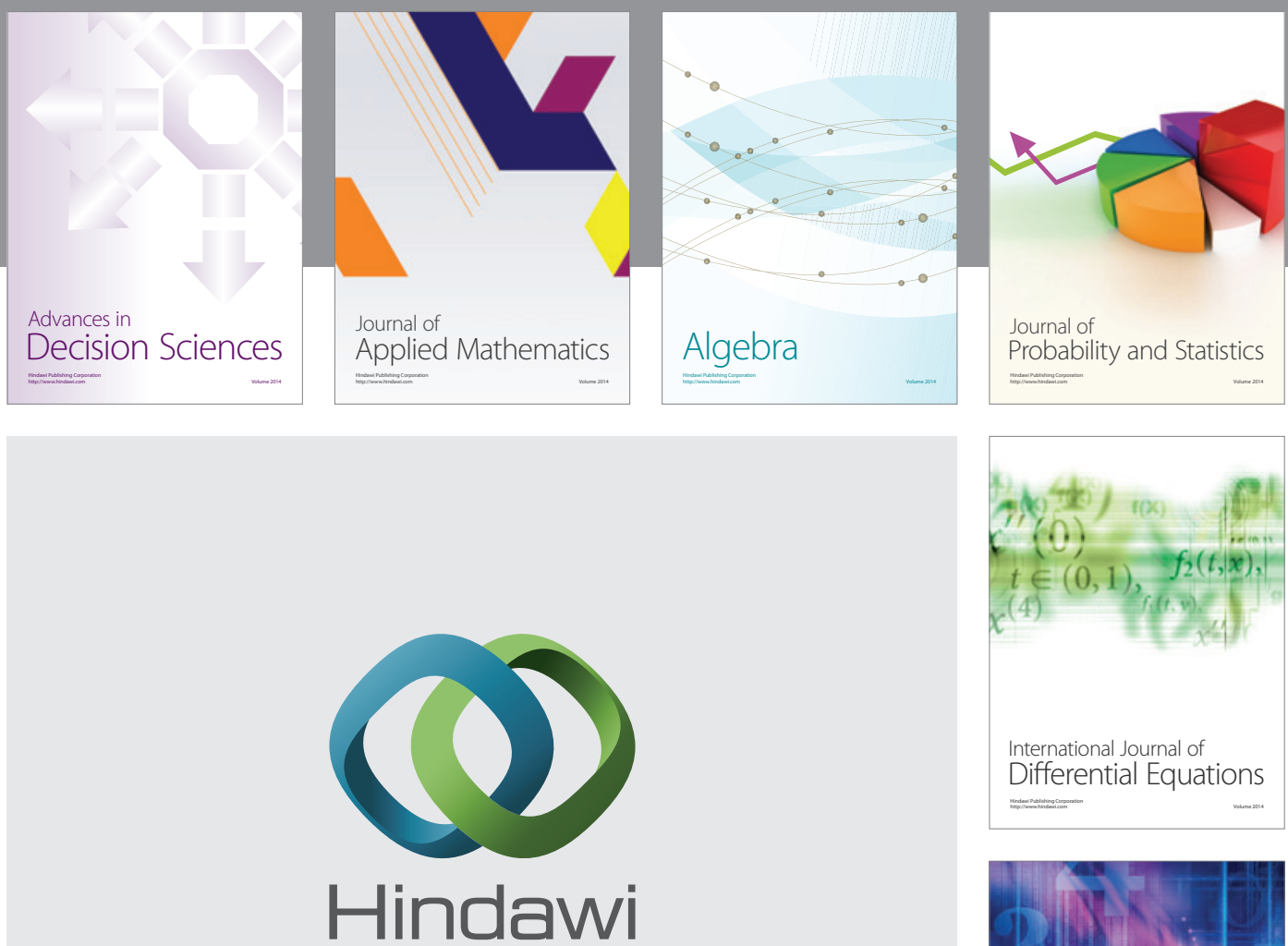

Submit your manuscripts at http://www.hindawi.com
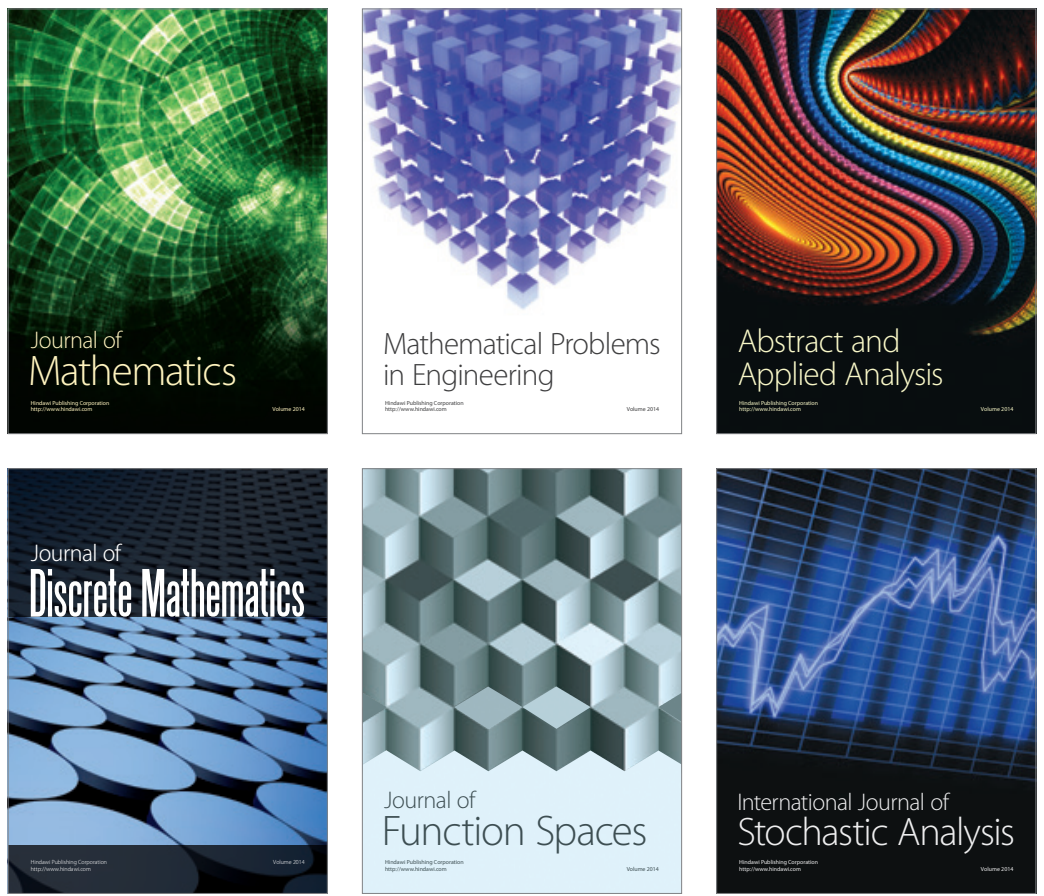

Journal of

Function Spaces

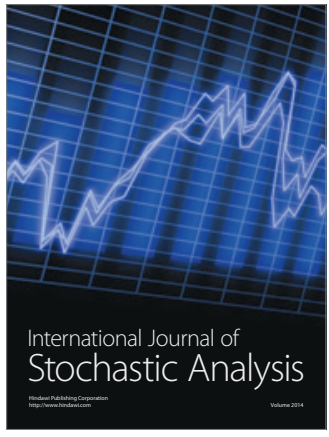

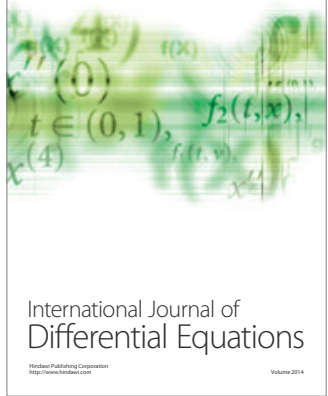
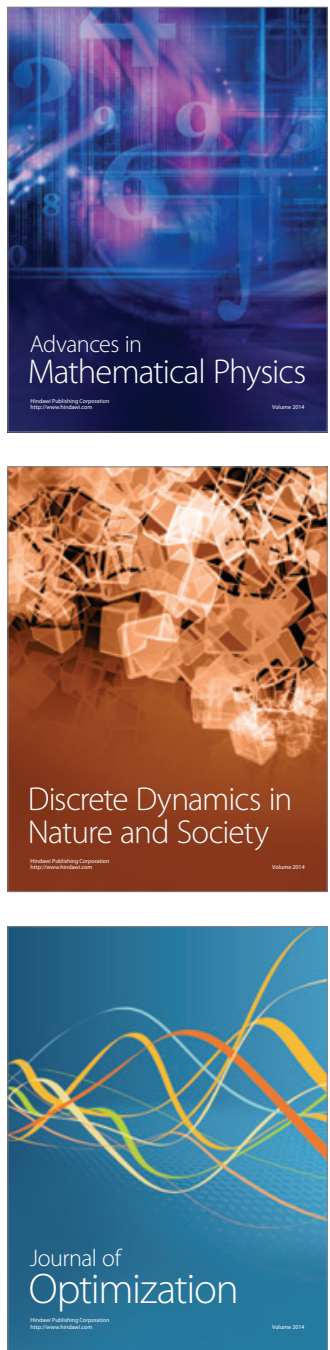